UCRL-ID-133244

\title{
A Source for Quantum Control: Generation and Measurement of Attosecond Ultraviolet Light Pulses
}

\author{
K. C. Kulander
}

February 19, 1999

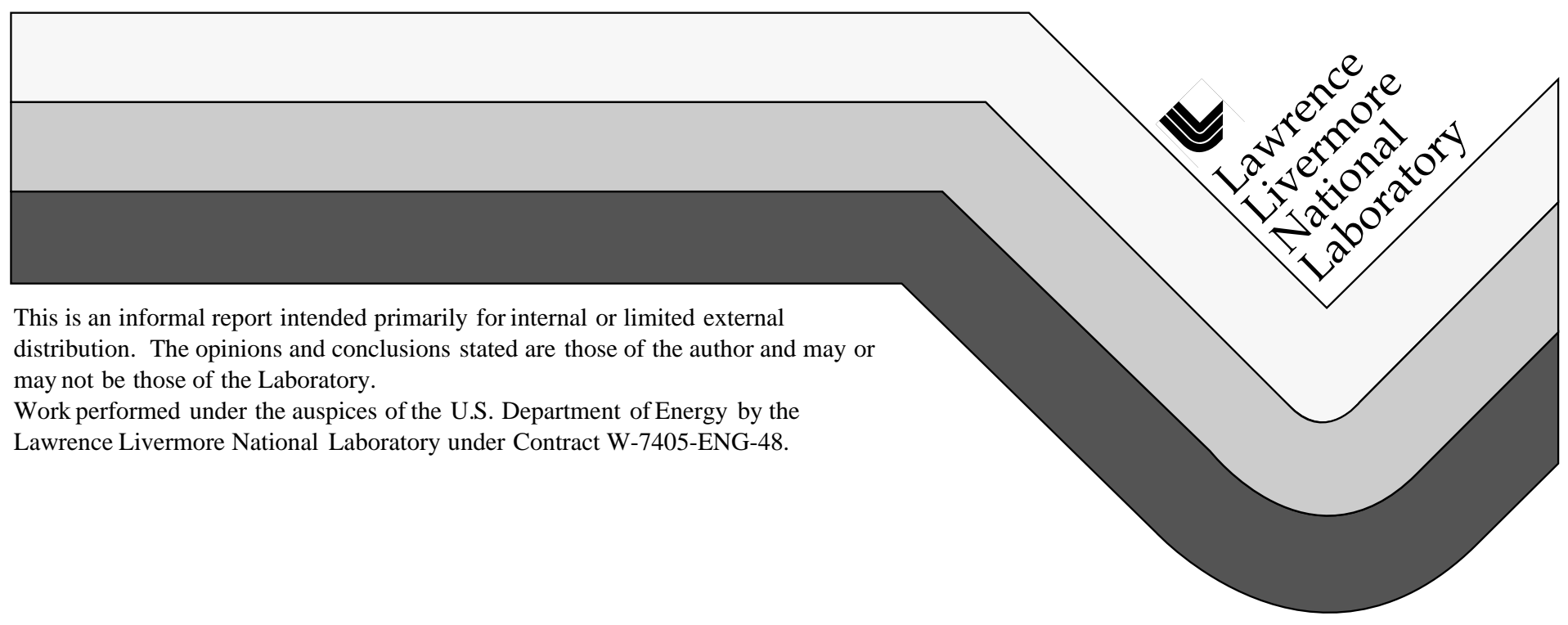




\section{DISCLAIMER}

This document was prepared as an account of work sponsored by an agency of the United States Government. Neither the United States Government nor the University of California nor any of their employees, makes any warranty, express or implied, or assumes any legal liability or responsibility for the accuracy, completeness, or usefulness of any information, apparatus, product, or process disclosed, or represents that its use would not infringe privately owned rights. Reference herein to any specific commercial product, process, or service by trade name, trademark, manufacturer, or otherwise, does not necessarily constitute or imply its endorsement, recommendation, or favoring by the United States Government or the University of California. The views and opinions of authors expressed herein do not necessarily state or reflect those of the United States Government or the University of California, and shall not be used for advertising or product endorsement purposes.

This report has been reproduced directly from the best available copy.

Available to DOE and DOE contractors from the Office of Scientific and Technical Information P.O. Box 62, Oak Ridge, TN 37831

Prices available from (423) 576-8401

Available to the public from the National Technical Information Service

U.S. Department of Commerce 5285 Port Royal Rd. Springfield, VA 22161 
A Source for Quantum Control: Generation and Measurement of Attosecond Ultraviolet Light Pulses

Kenneth C. Kulander, Principle Investigator

H Division, Physics Department

Lawrence Livermore National Laboratory

This project has pursued the possibility of producing ultra-short pulses of coherent light using harmonic conversion of a mid-infrared light source, focused into an atomic gas medium. This was a joint effort with Louis DiMauro's experimental group at Brookhaven National Laboratory and in collaboration with Ken Schafer from Louisiana State University and Mette Gaarde from Lund University on the theoretical part.

High order harmonic generation (HHG) in nobel gas media using short-pulse visible and near infrared lasers has become an established method for producing coherent, short pulse radiation at wavelengths from the ultraviolet to soft $x$-rays. We recently proposed that this approach could lead to extremely short pulses, potentially less than one fs, provided the unavoidable frequency chirp of the highest harmonics, could be removed by compressing the pulses with a grating pair. Sources of sub-fs pulses would provide unique opportunities to study dynamical processes on time scales short compared to those associated with nuclear motion. Truly stroboscopic pictures of chemical reaction dynamics would be possible, for example. In this research project we have chosen much smaller driving frequencies than used previously in HHG studies for two reasons. First, this will allow us to measure the pulse lengths of the compressed harmonics because they will be in the vacuum ultraviolet where coincidence measurements are possible. Second, the wavelengths of these harmonics will be idea for pump-probe experiments of quantum dynamical control studies.

Our theoretical effort was concentrated in two areas. We used our timedependent quantum numerical codes to evaluate the harmonic response of alkali atoms to the mid-IR laser excitation. Results were obtained for potassium, the initial species to be used in the experiments, then sodium and rubidium to investigate the possibility of higher conversion efficiencies. In fact, rubidium was found to be significantly better than potassium, both because it provided a stronger harmonic response and because the target gas could be maintained at about an order of magnitude higher pressure than with potassium. The second theoretical focus was the development of an adiabatic approximation for solving the equations governing the propagation of the generated harmonic fields through the laser-excited medium. A code was constructed using this approximation and the first phase matching 
calculations in this ultra-short pulse regime were carried out. Two papers have been written about this work and published in The Physical Review A.

The experimental effort was slowed by difficulties with the potassium source which were solved and photoelectron and harmonic spectra were obtained, the first ever at these wavelengths. These preliminary experiments were carried out using a ps laser source and an estimate of the bandwidth of the harmonics was obtained. The spectral width indicated that our prediction that the harmonic pulse lengths can be two orders of magnitude shorter than the driving field appears probable. Soon the experiments will switch to a $100 \mathrm{fs}$ driver and the attempt to reach the sub-fs regime will then be possible. We found that potassium, the target in the initial studies had a lower than expected conversion efficiency so that experiments were initiated using rubidium. This has proved to be better by more than three orders of magnitude. A large fraction of this improvement was due to the metal source running at a much higher pressure. A further increase will be obtained when the shorter pulse driver is installed. An additional paper on these preliminary results is now being prepared.

The project was quite successful, although a bit behind schedule because of experimental problems. The theoretical predictions seem to have been corroborated by the measurements thus far. We also found some unexpected differences in the behavior of the different alkali atoms to mulitphoton excitation and ionization at these wavelengths. LDRD funding has been obtained at Brookhaven to continue the work and it is anticipated the desired results will be obtained during the coming year.

Publications:

Gaarde, M. B.; Antoine, Ph.; L'Huillier, A.; Schafer, K. J. and Kulander, K. C., Macroscopic studies of short-pulse high-order harmonic generation using the time-dependent Schrödinger equation, Physical Review A 57, 4553 (1998) UCRL-JC-129924.

Gaarde, M. B.; Salin, F.; Constant, E.; Balcou, Ph.; Schafer, K. J.; Kulander, K. C. and L'Huillier, Spatio-temporal separation of high harmonic radiation into two quantum path components, Physical Review A 59, 1367 (1999)

UCRL-JC-131594. 